\title{
Observations and Modeling of the Climatic Impact of Land-Use Changes 2014
}

\author{
Xiangzheng Deng, ${ }^{1}$ R. B. Singh, ${ }^{2}$ Qun'ou Jiang, ${ }^{3}$ Jinwei Dong, ${ }^{4}$ and Hongbo $\mathrm{Su}^{5}$ \\ ${ }^{1}$ Institute of Geographic Sciences and Natural Resources Research, Chinese Academy of Sciences (CAS), Beijing 100101, China \\ ${ }^{2}$ Department of Geography, Delhi School of Economics, University of Delhi, Delhi 110007, India \\ ${ }^{3}$ School of Soil and Water Conservation, Beijing Forestry University, Beijing 100038, China \\ ${ }^{4}$ Department of Microbiology and Plant Biology, Center for Spatial Analysis, University of Oklahoma, Norman, OK 73019, USA \\ ${ }^{5}$ Department of Civil, Environmental and Geomatics Engineering, Florida Atlantic University, Boca Raton, FL 33431-0991, USA
}

Correspondence should be addressed to Xiangzheng Deng; dengxz.ccap@gmail.com

Received 4 May 2015; Accepted 4 May 2015

Copyright (C) 2015 Xiangzheng Deng et al. This is an open access article distributed under the Creative Commons Attribution License, which permits unrestricted use, distribution, and reproduction in any medium, provided the original work is properly cited.

Land Use and Cover Change (LUCC) is synthetically driven by a combination of natural and human induced factors including urbanization, grassland degradation, cultivated land reclamation, deforestation, and geological hazards. LUCC has been observed at different spatial and temporal scales in the context of regional or global climate changes. At the same time, LUCC can respond to and make an important impact on the global or regional climate changes. For example, LUCC directly changes the land surface conditions, such as albedo, roughness, and imperviousness, and indirectly changes the aerosol and compositions in the atmosphere, which in turn will change the energy balance and mass exchanges at the land and atmospheric interface. Study of the climatic impact of LUCC is of interest to both scientists and policy makers. Investigation of the interaction between LUCC and climate changes is a hot research topic in the scientific community, although it remains a challenging task because of the strong coupling between LUCC and climate changes. For example, to correctly interpret long term data records of surface temperature observations from meteorological stations, the site locations have to be distinguished between rural areas and urban areas. To improve our understanding of the climatic impact of LUCC, we need to develop more observation techniques, observation datasets, and modeling frameworks to support the data analysis, decision making of LUCC.

The studies included in this special issue cover diversified areas in LUCC and climate changes. The first category of the studies is on observation techniques and observation datasets related to LUCC and the climatic impact. Y. Li et al. observed the different impact of land use patterns on the evapotranspiration for a grassland ecosystem. B. Yang et al. studied the impact of body of water in a city on the heat island effect using satellite based land surface temperature observations. Their finding is, the area and spatial locations of the body of water can be optimized to maximize the cooling effects from the waters in urban areas. X. Che et al. developed a method to downscale the MODIS surface reflectance to improve the extraction of body of water. X. Yao et al. used the LUCC data in 20 years to study the impact on regional temperature and precipitation in the middle and lower reaches of the Yangtze River. It was revealed that the impact of LUCC on regional temperature is significant, but not on regional precipitation in this region. W. Hu et al. investigated the relationship between the land use intensity and the surface temperature changes in the Dongting Lake area, China, from year 2001 to year 2010 based on the datasets derived from Landsat TM. It was reported that the surface temperature was more sensitive to the land use intensity in low altitude areas than in high altitude areas.

The second category of studies in this special issue is on the modeling of LUCC and the climatic impact. M. Wang and $\mathrm{X}$. Yan used two methods named the "urban minus rural" and the "observation minus reanalysis" to quantify the potential impact of the urbanization in the BeijingTianjing-Hebei metropolitan area on the regional temperature changes. Seasonal patterns of the regional temperature changes were simulated and it was found out that the latter 
method performed relatively better in this case study. F. Wu et al. did scenario analysis of potential climate changes and LUCC in Heihe River Basin of China using the Soil and Water Assessment Tool (SWAT) and investigated the hydrological impact in different scenarios. Z. Wang et al. used a structural equation model to study the impact of LUCC on the regional climate in Southern China. F. Wu et al. used a hydrologic model and scenario analyses to quantify the impact of forest area changes in the head-water area of a river basin on the water yield variation. $\mathrm{Z}$. Li et al. used the binary panel logit regression to separate the impact of LUCC, economic development, and climate change on the land degradation represented by the NDVI.

This special issue also includes a category of studies on adaptation/management of LUCC and the regional climate changes. X. Mao et al. investigated the efficiency of using constructed wetlands to remove the pollutant from the agricultural wastewater. The seasonal variation of the purification efficiency was reported in their study. X. Deng et al. identified the water scarcity issue for an ecologically fragile area in a river basin in northwestern China and proposed solutions to adapt to the regional climate changes. W. Song et al. assessed the different impact of two vital ecological policies (Grain-for-Green and Grain-for-Blue) on the ecosystem service values based on the net primary productivity and soil erosion. D. Lin and Y. Lin used an empirical analysis to model the game situation and analyze the game behaviors of stakeholders of voluntary forest carbon offset projects in China. They concluded that a win-win solution can be achieved through the joint participation and a compromise in the voluntary forest carbon offset projects. C. Shi et al. developed a system to optimize the land use zoning to maximize the ecosystem service values under scenarios of LUCC and climate changes for the middle reaches of Heihe River Basin in North China. G. Jin et al. employed the fuzzy weight of evidence model to assess the land use suitability in a mountain area to avoid the potential geological hazards in a scenario of rapid urbanization in Yunan Province, China. D. Yuan et al. presented a method to establish the feasibility of Certified Emission Reduction (CER) quantitative evaluation, in order to implement the Clean Development Mechanism $(\mathrm{CDM})$ at the United Nations Framework Convention on Climate Change (UNFCCC).

All the papers in this special issue presented a collection of latest research results in the field of observations and modeling of the climatic impact of land-use changes. This special issue is intended to advance our understanding of the mechanisms and impacts of LUCC on climate change and even the human well-being. We are quite optimistic that these articles would be of great interest to the readers of this journal and will be of help to address the new emerging challenges in this field.

\section{Acknowledgments}

As guest editors of this special issue, we would like to thank all the authors for their contributions to this volume. Special mention of thanks and appreciation is due to the international panel of reviewers for making their expertise available for evaluation of the manuscripts.
Xiangzheng Deng

R. B. Singh

Qun'ou Jiang

Jinwei Dong

Hongbo Su 

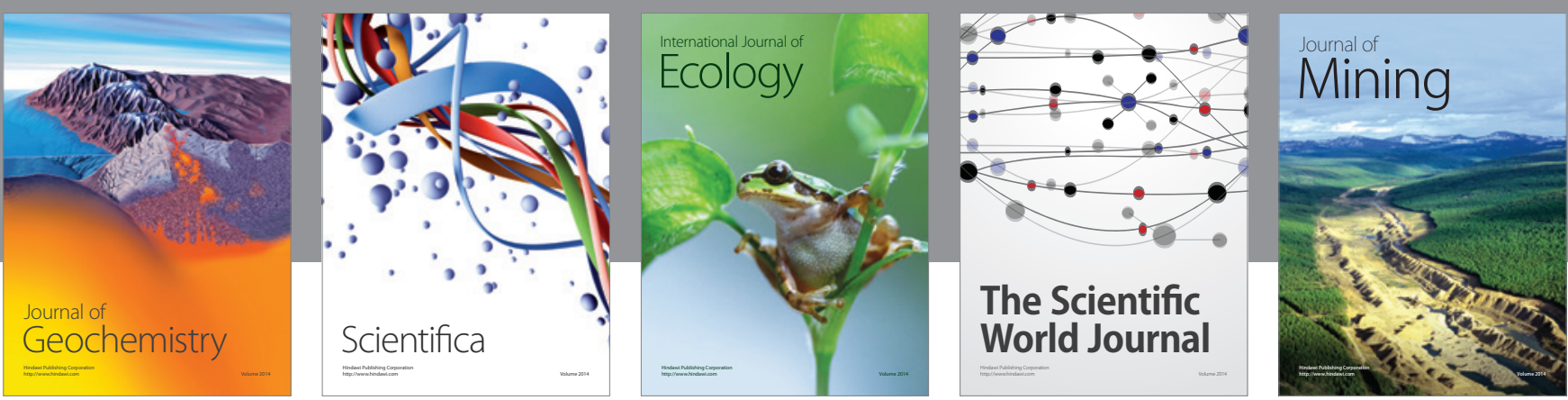

The Scientific World Journal
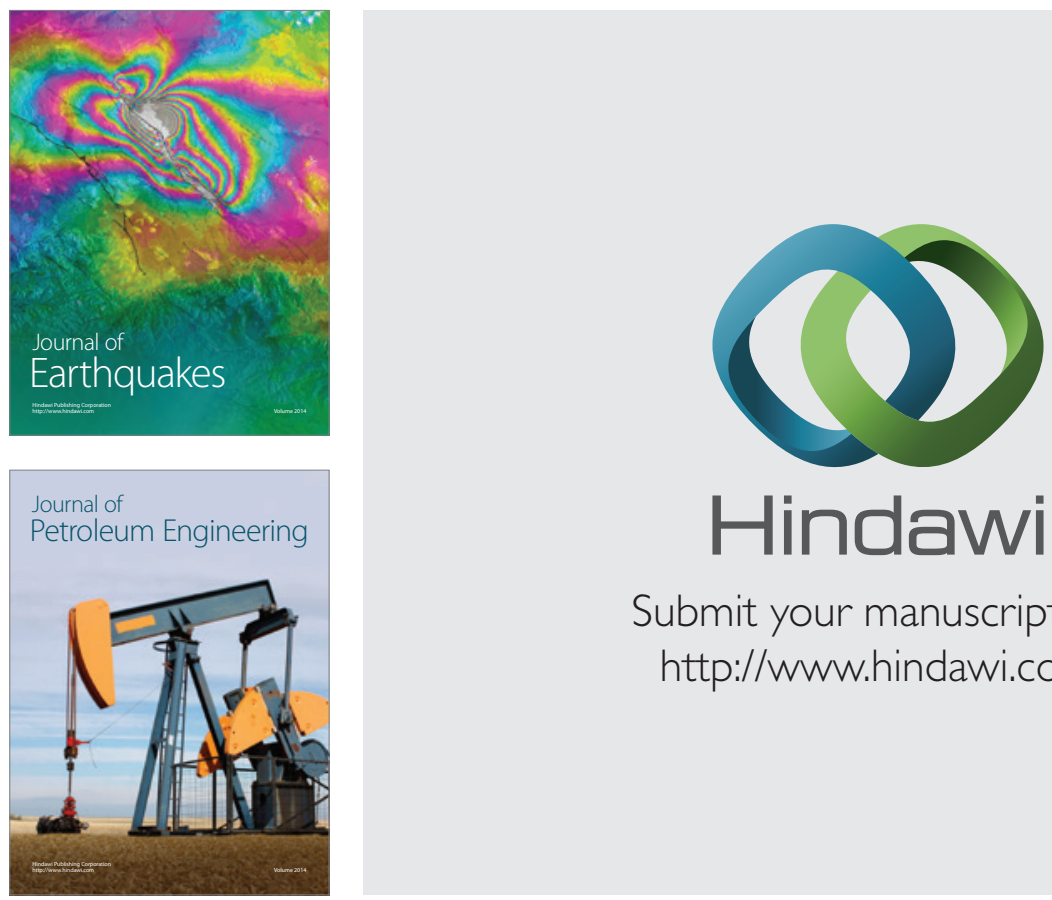

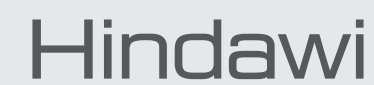

Submit your manuscripts at

http://www.hindawi.com
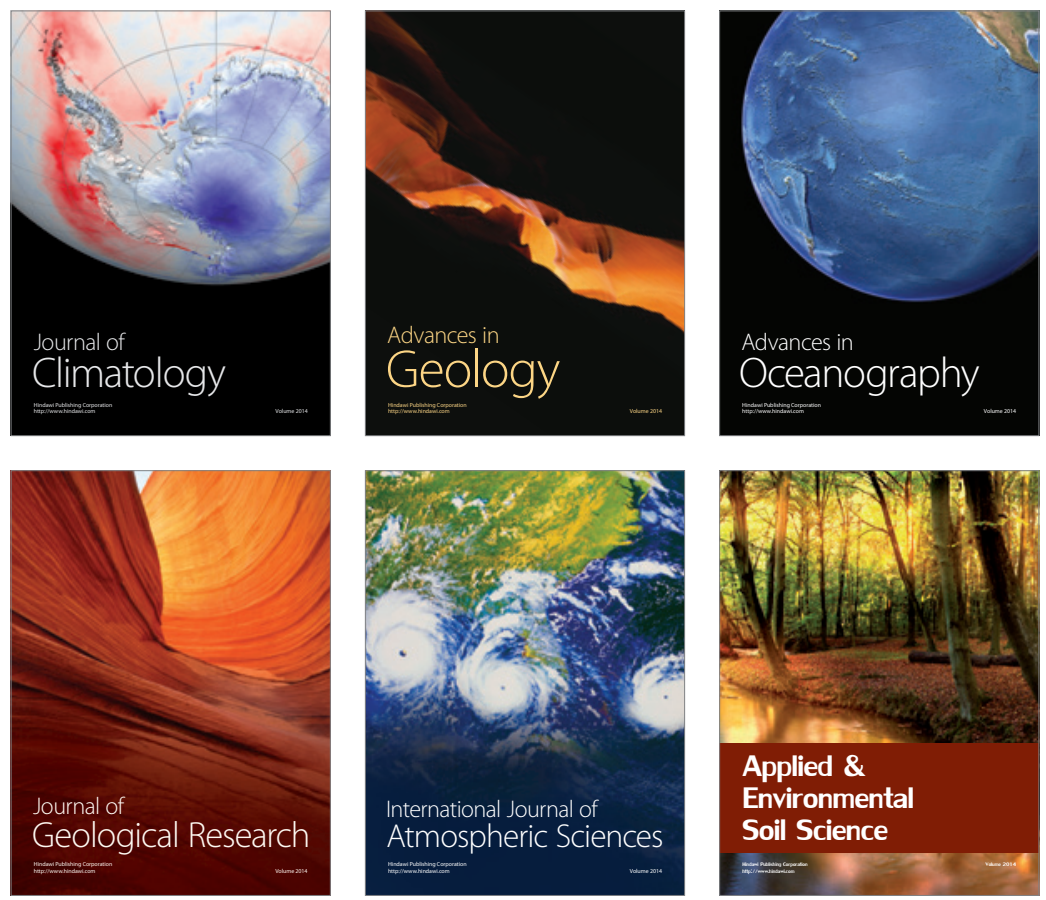
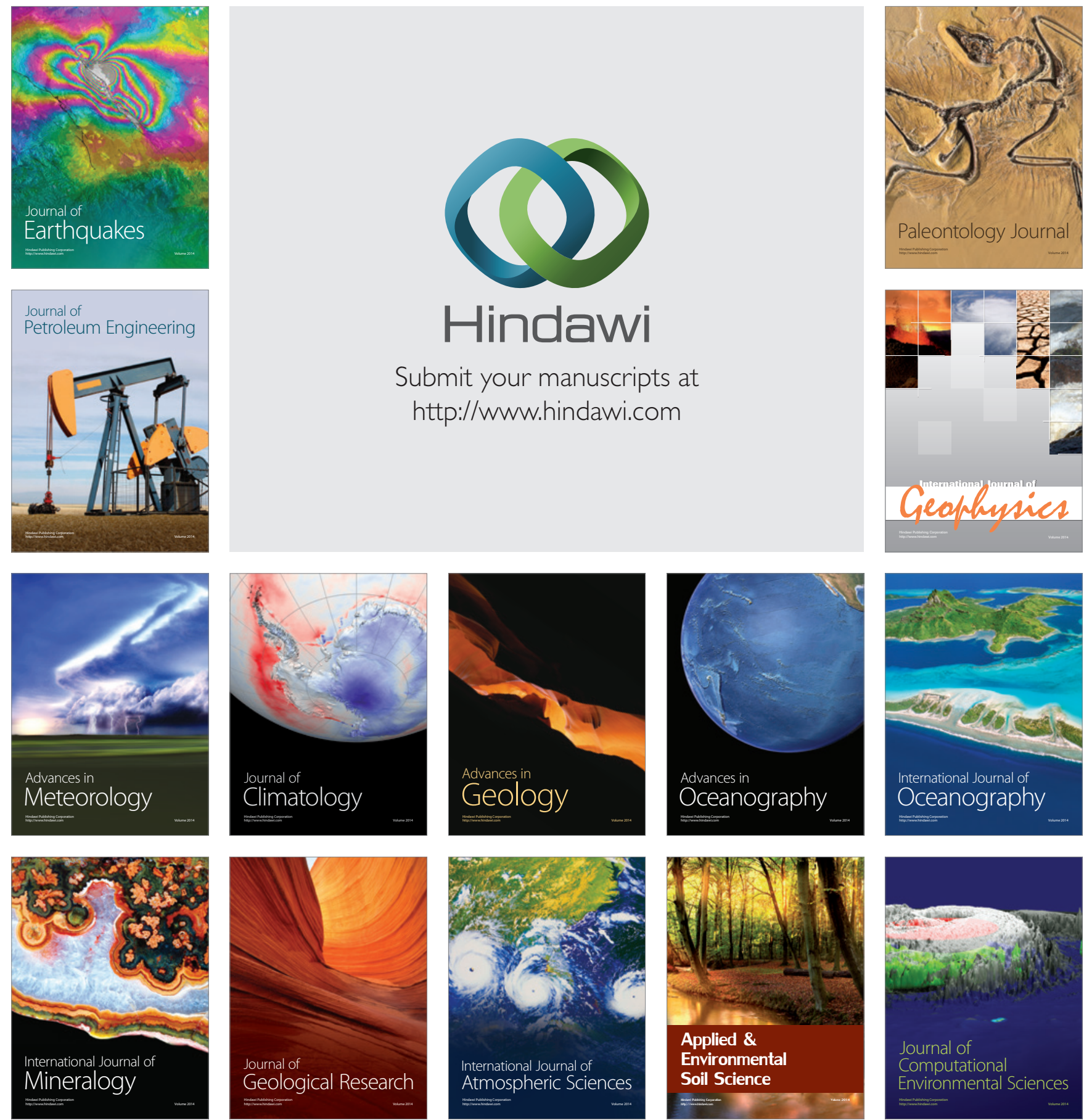